\title{
Receptivity of Swept-Wing Boundary Layers to Surface Roughness and Inhomogeneous Suction
}

\author{
Daniel Simanowitsch ${ }^{1,2(\bowtie)}$, Alexander Theiss ${ }^{1,2}$, and Stefan Hein ${ }^{1,2}$ \\ 1 Cluster of Excellence SEA - Sustainable and Energy-Efficient Aviation, \\ Technische Universität, Braunschweig, Germany \\ 2 Institute of Aerodynamics and Flow Technology, German Aerospace Center (DLR), \\ Bunsenstr. 10, 37073 Göttingen, Germany \\ \{daniel.simanowitsch, alexander.theiss, stefan.hein\}@dlr.de
}

\begin{abstract}
Suction through porous aircraft wings is a promising concept to reduce its drag, but the modified boundary layer can be more susceptible to external forcing, like surface roughness and inhomogeneities in the suction distribution. This paper analyzes the effects of suction on the linear receptivity of a swept-wing boundary layer to surface roughness and non-uniform suction, focusing on stationary crossflow instabilities. Employing a compressible adjoint approach, receptivity coefficients for a Falkner-Skan-Cooke similarity solution and a transonic swept-wing boundary layer on an A320 vertical tail plane with and without suction are given.
\end{abstract}

Keywords: Receptivity $\cdot$ Parabolized stability equations $\cdot$ Adjoint

\section{Introduction}

Aerodynamic drag has a significant influence on the fuel consumption of modern airplanes. Hybrid Laminar Flow Control (HLFC) is a promising concept to reduce the drag of future commercial airplanes. Suction through porous surfaces is used to delay laminar-turbulent transition and thus to reduce the overall skin-friction drag. In three-dimensional boundary layers (BL), suction in the leading-edge region of the transonic swept wings is used to stabilize crossflow (CF) instabilities. The thinner BL can be more susceptible to a given external forcing, however, i.e. the effectiveness with which $\mathrm{CF}$ instabilities are generated by environmental disturbances increases. Moreover, suction through porous surfaces typically increases surface roughness and results in a non-uniform suction distribution. Both types of external forcing can trigger stationary CF instabilities. In $N$-factor based transition prediction with Linear Stability Theory (LST) or with Parabolized Stability Equations (PSE), the processes that lead to the formation of boundary-layer instabilities are not considered at all. Hence, only the stabilizing effect of the idealized spanwise homogeneous suction on the subsequent development of the boundary-layer instabilities is covered. Changes in

(C) The Author(s), under exclusive license to Springer Nature Switzerland AG 2021

A. Dillmann et al. (Eds.): STAB/DGLR Symposium 2020, NNFM 151, pp. 224-234, 2021.

https://doi.org/10.1007/978-3-030-79561-0_22 
the receptivity characteristics of the modified boundary-layer flow and the typically increased external forcing due to suction through porous surfaces, which presumably both contribute to the increase in initial boundary-layer instability amplitudes, therefore have to be compensated by assuming lower CF transition $N$-factors when predicting laminar-turbulent transition on HLFC wings.

Receptivity coefficients are a local measure quantifying the effectiveness with which a boundary-layer instability mode can be generated by a certain type of external forcing. Only few data exist in literature on receptivity coefficients of realistic transonic swept-wing boundary layers without and with suction. Together with information on the actual surface roughness and suction distribution, such data allow quantifying detrimental effects on the emergence of instabilities and could provide guidelines on how to adjust the corresponding transition $\mathrm{N}$-factors. The negative impact of suction in boundary layers on receptivity has been shown for Tollmien-Schlichting waves in a two-dimensional boundary layer by Crouch [1]. Janke [2] has previously shown that an equivalent forcing model together with a PSE-like method is a suitable tool to predict receptivity of threedimensional boundary layers to roughness and suction by comparing the results to a Direct Numerical Simulation (DNS). An efficient approach for receptivity modeling, which does not require a new direct solution for each forcing configuration, is to employ the adjoint solution. How adjoint Orr-Sommerfeld and PSE equations predict forced and natural receptivity has been extensively studied by Hill [3,4]. Adjoint solutions have henceforth been employed to e.g. determine sensitivities of BL flows [5], receptivity to roughness, suction, acoustic and freestream disturbances [6,7]. Schrader [8] employed a DNS solution to determine receptivity coefficients for the self-similar Falkner-Skan-Cooke (FSC) boundary layer, while Piot and Schrader [9] expanded his work and showed that these coefficients are dependent on the forcing shape in spatially evolving flows, when the Fourier component of the shape at the chordwise instability wavenumber is small. Bertolotti [10] suggested to include the streamwise derivative of the shape function as a remedy.

This paper demonstrates the influence of suction on the local boundarylayer receptivity to surface roughness and inhomogeneous suction. We focus on steady crossflow instabilities in swept-wing boundary layers. Compressible PSE or LST are used to quantify the downstream growth of instabilities and adjoint PSE or LST respectively are employed to quantify receptivity. This approach is validated for a Falkner-Skan-Cooke BL and applied to the boundary layer on an A320 vertical tail plane. The flow over this fin is analyzed with and without a generic suction distribution, and the receptivity coefficients for both cases are given.

\section{Methodology}

In this section, the equations to obtain the linear receptivity coefficients for infinite swept-wing flows are derived. A compressible, non-local formulation for the direct and adjoint problem in curvilinear coordinates is chosen to analyze realistic airfoil flows. 


\subsection{PSE}

To derive the compressible Parabolized Stability Equations (PSE), the total three-dimensional flow is split into the mean flow quantities $Q=\{\bar{\rho}, U, V, W, \bar{T}\}$ and disturbance quantities $q^{\prime}=\left\{\rho^{\prime}, u^{\prime}, v^{\prime}, w^{\prime}, T^{\prime}\right\}$, which are assumed to be small. In the following, $\{\mathrm{x}, \mathrm{y}, \mathrm{z}\}$ denote the chordwise, wall-normal and spanwise directions respectively and $\{\mathrm{U}, \mathrm{V}, \mathrm{W}\}$ the respective velocities. The non-dimensional, compressible Navier-Stokes equations are then formulated in disturbance form by introducing this decomposition and subtracting the equations describing the baseflow (see [6]). The PSE equations are then derived from a Wentzel-KramersBrillouin (WKB) ansatz where the disturbance $q^{\prime}$ is decomposed into a shape function $q$ and a phase function $\Theta$ :

$$
\begin{aligned}
q^{\prime}(x, y, z, t)= & q(x, y) \Theta(x) \mathrm{e}^{i \beta z-i \omega t}, \\
& \Theta(x)=\mathrm{e}^{i \int_{x_{0}}^{x} \alpha\left(x^{\prime}\right) \mathrm{d} x^{\prime}}
\end{aligned}
$$

Hereby $q=\{\rho, u, v, w, T\}$ is assumed to be slowly varying in chordwise direction $x$ and $\hat{q}=q \Theta$. An ambiguity arises due to both shape function and exponential part being functions of $x$, which is removed with the condition $\int_{0}^{\infty} q^{H} \frac{1}{h_{1}} \frac{\partial q}{\partial x} h_{2} \mathrm{~d} y=0$, ensuring minimal streamwise changes of $q$ across $y$. The scale factors $h_{i}$ are necessary for a derivation in an arbitrary orthogonal curvilinear coordinate system. Ansatz 1 is then introduced into the linearised Navier-Stokes equations, a scale separation between slow variations in chordwise direction and faster ones in wall-normal direction is performed, resulting in a parabolic equation system of the form $L_{p} q=0$, with $L_{p}$ the linear operator defined as follows

$$
L_{p}=A+B \mathfrak{D}_{y}+C \mathfrak{D}_{y y}+D \mathfrak{D}_{x},
$$

with

$$
\mathfrak{D}_{x}=\frac{1}{h_{1}} \frac{\partial}{\partial x}, \quad \mathfrak{D}_{y}=\frac{1}{h_{2}} \frac{\partial}{\partial y}, \quad \mathfrak{D}_{y y}=\frac{1}{h_{2}^{2}} \frac{\partial^{2}}{(\partial y)^{2}} .
$$

The operator matrices $A, B, C$ and $D$ can be found in [11]. These equations can be solved marching downstream with boundary conditions

$$
T=u=v=w=0 \quad \text { at } \quad y=0 \text { and as } \quad y \rightarrow \infty .
$$

The equation system of LST can be derived from Eq. (2) by introducing the assumption of locally parallel flow and neglecting non-local terms, i.e. $D=0$.

\subsection{Roughness and Inhomogeneous Suction Boundary Conditions}

The surface roughness is not simulated directly, but instead modeled via inhomogeneous boundary conditions [8]. No-slip conditions are projected using a Taylor-series expansion under the assumption that the roughness height is small 
compared to the boundary-layer thickness, thus truncating it at first order. Therefore, the boundary conditions are:

$$
\begin{aligned}
& \hat{u}(x, 0)=-h_{x} \frac{\partial U}{\partial y}, \quad \hat{v}(x, 0)=-h_{x} \frac{\partial V}{\partial y}, \\
& \hat{w}(x, 0)=-h_{x} \frac{\partial W}{\partial y}, \quad \hat{T}(x, 0)=-h_{x} \frac{\partial \bar{T}}{\partial y},
\end{aligned}
$$

with some spanwise periodic roughness element $h(x, z)=h_{x}(x) \cdot \mathrm{e}^{i \beta_{R} z}, \beta_{R}$ its spanwise wavenumber and $h_{x}(x)$ the chordwise shape function. A roughness with shape $h$ triggers instabilities with the same spanwise wavenumber $\beta$.

Analogously, an inhomogeneous suction distribution $v_{s}(x, z)=v_{b}(x) \cdot \mathrm{e}^{i \beta_{S} z}$ with the spanwise wavenumber $\beta_{S}=\beta$ is defined on top of an arbitrary spanwise homogeneous distribution. The difference to the homogeneous boundary conditions (BC) in Eq. (4) is:

$$
\hat{v}(x, 0)=v_{b}
$$

\subsection{Adjoint PSE}

Knowledge of the initial amplitudes of any disturbance inside the boundary layer is necessary for a precise assessment of the transition location. Receptivity analysis via adjoint equations aims to provide these amplitudes effectively.

The derivation employs a definition for the inner product ' $<\cdot, \cdot>$ ':

$$
<a, b>=\int_{X_{0}}^{X_{1}} \int_{0}^{\infty} a^{H} b h_{1} h_{2} \mathrm{~d} x \mathrm{~d} y
$$

with ()$^{H}$ being the conjugate transpose. We define an adjoint operator $L_{p}^{*}$ and obtain the following Lagrange identity

$$
<q^{*}, L_{p} q>=<L_{p}^{*} q^{*}, q>+\int_{X_{0}}^{X_{1}} \int_{0}^{\infty} \nabla \cdot J\left(q, q^{*}\right) h_{1} h_{2} \mathrm{~d} x \mathrm{~d} y
$$

with the adjoint flow vector $q^{*}=\left\{\rho^{*}, u^{*}, v^{*}, w^{*}, T^{*}\right\}$ and the bilinear concomitant $J=\left(J_{x}, J_{y}\right)^{T}$. Since the adjoint quantities $q^{*}$ can be chosen arbitrarily, we define them to fulfill $L_{p}^{*} q^{*}=0$. After partial integration of the left side in Eq. (8), the adjoint operator is derived as:

$$
L_{p}^{*}=A^{*}+B^{*} \mathfrak{D}_{y}+C^{*} \mathfrak{D}_{y y}+D^{*} \mathfrak{D}_{x} .
$$

The adjoint operators $A^{*}, B^{*}, C^{*}$ and $D^{*}$ can be found in Ref. [5]. Again, the equation system of adjoint LST can be derived from Eq. (9) by introducing the assumption of locally parallel flow and neglecting non-local terms, i.e. the $D^{*}$-term is zero.

If no external disturbances are introduced inside the domain, the absolute value of the streamwise part of the integrated bilinear concomitant $\int_{0}^{\infty} J_{x} h_{2} \mathrm{~d} y$ 
is a constant and therefore poses a natural normalisation value, which is also derived from the integration by parts as:

$$
J_{x}=q^{* H} D q
$$

with $q^{*}$ and $q$ obtained from the unforced problem with boundary conditions Eq. (4).

\subsection{Receptivity Coefficients}

To calculate amplitudes triggered inside the boundary layers by external disturbances, the Lagrange identity Eq. (8) is expanded:

$$
\begin{gathered}
0=\int_{0}^{\infty}\left[J_{x}\right]_{x_{0}}^{x_{1}} h_{2} \mathrm{~d} y-\int_{x_{0}}^{x_{1}}\left[q^{* H}\left(B-\frac{1}{h_{1} h_{2}} \frac{\partial h_{1}}{\partial y} C-\mathfrak{D}_{y}(C)\right) q\right. \\
\left.-\mathfrak{D}_{y}\left(q^{* H}\right) C q+q^{* H} C \mathfrak{D}_{y}(q)\right]_{y=0}^{\infty} h_{1} \mathrm{~d} x
\end{gathered}
$$

Equation 11 offers an efficient way to calculate instability amplitudes from the adjoint solution. We define the complex instability amplitude $A$ as the streamwise disturbance velocity at the position $y_{\max }$ where its absolute value $|u|$ is maximal, $A(x)=\hat{u}\left(x, y_{\max }\right)$. To introduce $A$ into Eq. (11), a new variable $\hat{J}$ is defined, $\hat{J}=\int_{0}^{\infty} J_{x} h_{2} \mathrm{~d} y / A$. No incoming disturbances are assumed, i.e. $x_{0}$ is located upstream of any sources, thus $A\left(x_{0}\right)=0$. Equation 11 is simplified with the boundary conditions $\left(u^{*}, v^{*}, w^{*}, T^{*}\right)=0$ at $y=0$ and as $y \rightarrow 0$, and taking into account the entries of matrix $C$ (cf. [5]), Eq. (11) becomes

$$
A\left(x_{1}\right)=\frac{1}{\hat{J}\left(x_{1}\right)} \int_{x_{0}}^{x_{1}}\left[q^{* H} B q-q^{* H} \frac{1}{h_{1} h_{2}} \frac{\partial h_{1}}{\partial y} C q-\mathfrak{D}_{y}\left(q^{* H}\right) C q\right]_{y=0} h_{1} \mathrm{~d} x
$$

For the two cases considered in this work, surface roughness and inhomogeneous suction, we assume that $\hat{q}=q \Theta$ satisfies the boundary conditions Eq. (5) or Eq. (6) respectively. The receptivity coefficient $c_{s}(x ; \beta)$ for suction is then defined as

$$
c_{s}=\frac{1}{\hat{J}(x)}\left[q^{* H} B \tilde{q}-q^{* H} \frac{1}{h_{1} h_{2}} \frac{\partial h_{1}}{\partial y} C \tilde{q}-\mathfrak{D}_{y}\left(q^{* H}\right) C \tilde{q}\right]_{y=0} .
$$

and the receptivity coefficient $c_{r}(x ; \beta)$ for roughness as

$$
c_{r}=\frac{1}{\hat{J}(x)}\left[q^{* H} \frac{1}{h_{1} h_{2}} \frac{\partial h_{1}}{\partial y} C \tilde{q}+\mathfrak{D}_{y}\left(q^{* H}\right) C \tilde{q}\right]_{y=0}
$$

since inserting the boundary conditions Eq. (5) for $q=\Theta^{-1} \hat{q}$ into Eq. (12) results in the $B$-term vanishing. The receptivity coefficients $c_{s}$ and $c_{r}$ are formulated to be independent of the disturbance distribution at the wall (i.e. $v_{b}(x)$ or $h_{x}(x)$ ) 
by dividing Eq. (5) and Eq. (6) with the respective disturbance shape (e.g. $\tilde{u}=$ $\left.\hat{u} /\left(\Theta h_{x}\right)\right)$. The amplitude at some position $x_{1}$ is then calculated as

$$
A\left(x_{1}\right)=\int_{x_{0}}^{x_{1}} c_{i} f(x) h_{1} \mathrm{~d} x
$$

with $c_{i}$ being $c_{r}$ or $c_{s}$ and $f(x)$ being either $h_{x}$ or $v_{b}$ for a roughness or suction distribution respectively. Note that $c_{r}$ and $c_{s}$ have different units and can be compared in terms of amplitude $A$ if information on $f(x)$ is given.

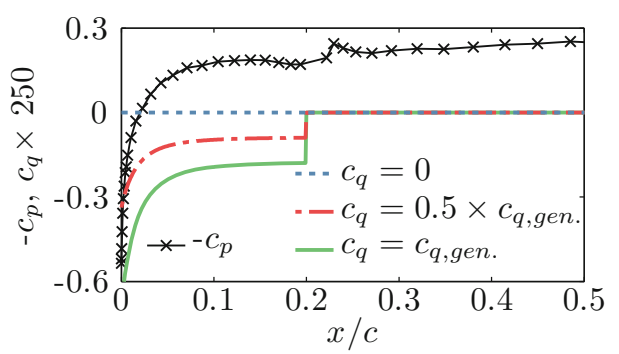

Fig. 1. Pressure and generic suction distributions.

\subsection{Baseflow Configuration}

The adjoint approach has been employed for the BL on the vertical tail plane (VTP) of an A320 aircraft. The flow data stem from a check-out flight test performed in November 2017 within the AFLoNext project [12]. The suction panel was located on both sides of the VTP, covering the first $20 \%$ of the chord length, designed as a double skin structure with a micro-perforated titanium sheet as outer skin supported by an inner sheet with stringers between them [12].

The laminar baseflow for the pressure distribution (Fig. 1) on the starboard side measured at a freestream Mach number of 0.76 and a side slip angle of $0.5^{\circ}$ was computed using the boundary-layer code COCO [13]. For the present study, the complicated suction distribution from the actual suction chamber design (see [12]) was replaced by a generic one, $c_{q, \text { gen }}$, resembling the actual distribution in terms of the level of suction and its chordwise evolution. The generic distribution is defined as

$$
c_{q, \text { gen }}=\frac{v_{b}}{Q_{\infty}}=-0.0007-0.002 \cdot(1+20 x / c)^{-3} \quad \text { for } \quad x / c<0.2
$$

with $Q_{\infty}$ being the freestream velocity and $c$ the chord length. Three baseflows have been generated by introducing multiples of $c_{q \text {,gen }}$ into COCO: (i) no suction, $c_{q}=0$, (ii) half suction, $c_{q}=0.5 \times c_{q \text {,gen }}$, and (iii) full suction, $c_{q}=c_{q, \text { gen }}$. 


\section{Results}

The receptivity coefficients are presented for a FSC similarity solution, verifying our implementation based on the adjoint LST and PSE framework, and for the realistic BL over an A320 vertical tail plane equipped with a suction panel.

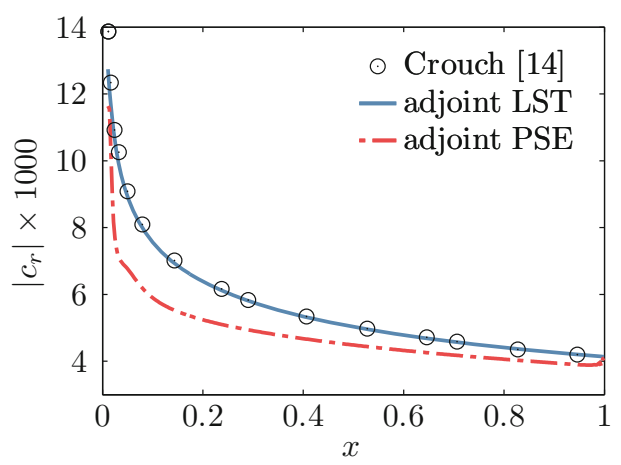

Fig. 2. Roughness receptivity coefficients for a FSC boundary layer.

\subsection{FSC Results}

The parameters of the FSC test case have been chosen as in [14], i.e. $\beta_{H}=0.6$ and $\phi_{n}\left(x_{n}\right)=30^{\circ}$ at $\operatorname{Re}\left(x_{n}\right)=1000$, with the Hartree parameter $\beta_{H}$ determining the pressure gradient and $\phi_{n}\left(x_{n}\right)$ denoting the local sweep angle at the normalization position $x_{n}$. We validate the results by following the adjoint method under the assumption of locally parallel flow, similar to [14]. The receptivity coefficients for a steady crossflow instability with a spanwise wavenumber of $\beta=0.45$ have been obtained utilizing compressible equations according to Eq. (13). The results are shown in Fig. 2 together with those in [14], derived from an incompressible set of equations. The results with parallel flow approximation are in perfect agreement, as compressibility has a negligible influence for this flow. As expected, receptivity is higher towards the leading edge and decreases further downstream, with maximum receptivity at the initial chordwise position. The PSE results show the influence of non-local and non-parallel effects. At larger values of $x$, the coefficients get similar to those obtained assuming parallel flow, while the discrepancy towards the leading edge is of the order of magnitude of $c_{r}$. Hence, local theory over-predicts receptivity to surface roughness.

Note that the depicted adjoint PSE results have also been compared to unpublished receptivity coefficients obtained from a different PSE code within the Telfona project [15]. Those non-local results also show a very good agreement. 


\subsection{A320 VTP Receptivity Results}

Since the flow conditions around the VTP are very similar for the port and starboard side, the following results are limited to the spatial extent of the suction panel of the VTP's starboard side. Figure 3 shows the $N$-factor results, calculated according to the local stability theory, for stationary crossflow instabilities for the case without suction (Fig. 3(a)) and the case with half of the generic suction rate (Fig. 3(b)). The $N$-factor is a measure of the accumulated growth of a disturbance and is defined as $N(x)=\ln \left(A(x) / A_{0}\right)$, where $A_{0}$ corresponds to the disturbance amplitude at the streamwise location where the instability starts to grow. For the case without suction, boundary-layer transition is expected at $x / c=0.016$, assuming a typical transition $N$-factor of 9 . In contrast, with only half of the generic suction rate, the overall growth of stationary crossflow instabilities (CFI) is probably insufficient to cause transition up to $x / c=0.2$. At the full generic suction rate, there is no amplification of stationary crossflow vortices on the suction panel anymore. Taking the surface curvature of the airfoil into account via metric terms in the local instability analysis, the maximum achievable $N$-factor reduces significantly, and at $c_{q}=0.5 \times c_{q, \text { gen. }}$, all stationary CFI are also damped. In the following, the receptivity investigations are limited to surface roughness and suction distributions with a spanwise wavenumber of $\beta=16001 / \mathrm{m}$. The CFI triggered at this wavenumber reaches one of the highest $N$-factors at the end of the suction panel (cf. Fig. 3(a)). Figure 4(a) depicts the receptivity of the boundary layer to surface roughness, whereas Fig. 4(b) shows the respective coefficient for non-uniform suction, both computed employing the adjoint LST framework. For all investigated cases, the receptivity coefficient for both roughness and inhomogeneous suction is highest in the leading-edge area and decreases towards the end of the suction panel. Note that the observable kink in the $c_{r}$-distribution at $x / c>0.15$ coincides with the beginning of an adverse pressure gradient region (cf. Fig. 1). In line with the expected trend, the boundary-layer thickness decreases with increasing suction rate and the flow becomes more receptive to disturbances at the wall. Hence, special care should
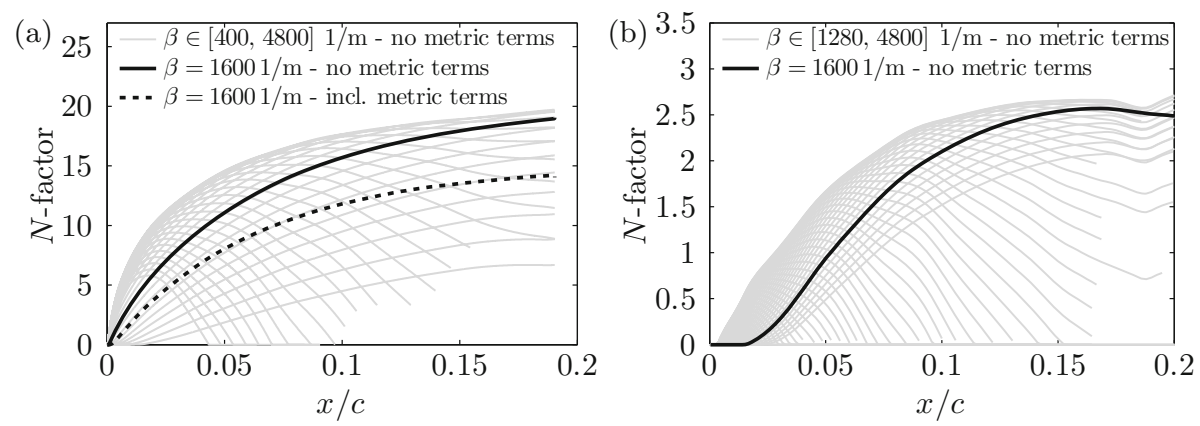

Fig. 3. Streamwise evolution of the $N$-factor for the stationary crossflow instability for selected spanwise wavenumbers $\beta$ in case of (a) $c_{q}=0$ and (b) $c_{q}=0.5 \times c_{q, g e n .}$. 

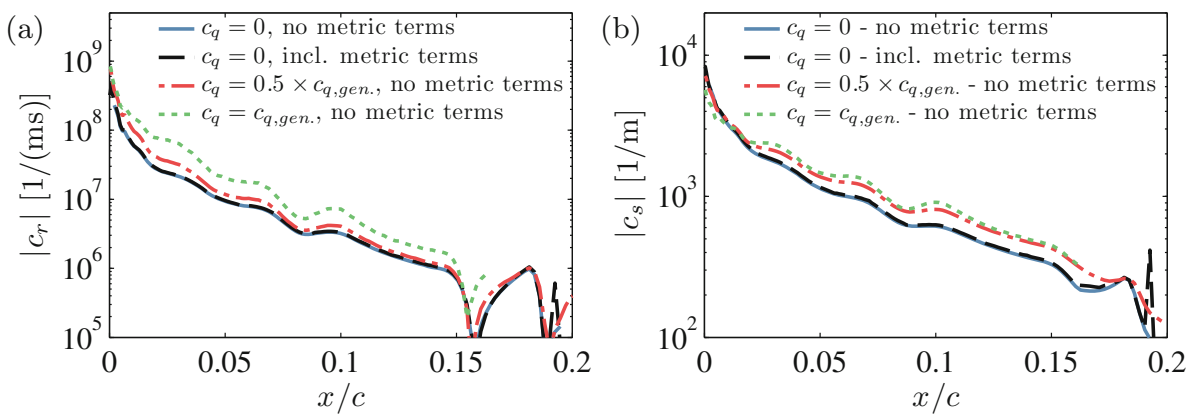

Fig. 4. Impact of the suction rate $c_{q}$ on the boundary-layer receptivity due to (a) surface roughness and (b) suction with $\beta=16001 / \mathrm{m}$.

be taken when arranging and manufacturing the suction panel to ensure that the desired effect of transition delay is not jeopardized by the increased receptivity efficiency of crossflow-dominated boundary layers to external forcing. The inclusion of the metric terms, which significantly impact the CFI growth rates (cf. Fig. 3(a)), affects the receptivity coefficients only to a small extent, as indicated by the black dashed line for the $c_{q}=0$ case in Fig. 4 .

Investigations utilizing the adjoint PSE framework are planned to further investigate the effects of nonlocal and non-parallel flow terms on the observed trends.

\section{Summary and Conclusions}

The impact of varying suction rate on the receptivity of crossflow-dominated boundary layers to surface roughness and non-uniform suction was studied. The corresponding receptivity coefficients were derived from direct and adjoint solutions of the linear local and nonlocal stability equations. The numerical approach was verified by comparing the roughness receptivity coefficients for a Falkner-Skan-Cooke similarity solution with suitable reference data from the literature. Subsequently, the receptivity analysis was extended to a realistic transonic swept-wing boundary-layer flow on the A320 vertical tale plane with a porous suction panel. The amount of suction was systematically varied starting from a generic chordwise suction distribution that resembles the suction distribution of the corresponding flight test data point.

It has been shown that for stationary crossflow vortices, the effects of surface curvature on the receptivity coefficients were negligible, in contrast to their significance for the crossflow disturbance amplification rates. Moreover, as boundary-layer thickness decreases with rising spanwise homogeneous suction rate, the boundary layer can be more susceptible to a given external forcing, like surface roughness and spanwise suction non-uniformities, which both can trigger stationary crossflow vortices. The results from the present local linear 
receptivity analysis confirm this expected trend and, for the first time, provided quantitative data on the rise of the corresponding linear receptivity coefficients with increasing mean suction rates for a realistic HLFC configuration. The rising receptivity of the boundary layer with suction is one of the two main reasons why typically lower $\mathrm{CF}$ transition $N$-factors have to be assumed in laminarturbulent transition prediction on HLFC wings. As the same external forcing leads to higher initial amplitudes when receptivity increases, the $N$-factor at a similiar transition amplitude decreases. The second contribution stems from the increased amount of external forcing due to the presence of the porous suction panel itself. Quantifying the latter effect would require detailed information on the corresponding changes in surface roughness and the suction non-uniformity, however.

Acknowledgments. We would like to acknowledge the funding by the Deutsche Forschungsgemeinschaft (DFG, German Research Foundation) under Germany's Excellence Strategy - EXC 2163/1 - Sustainable and Energy Efficient Aviation - Project-ID 390881007. The authors also express their thanks to Heiko von Geyr (DLR) for providing the A320 flight test data. The flight test activities received funding from the European Community's Seventh Framework Programme FP7/2007-2013, under grant agreement No. 604013, AFLoNext project.

\section{References}

1. Crouch, J. D.: Receptivity issues for laminar-flow control. In: IUTAM Symposium on Mechanics of Passive and Active Flow Control. Fluid Mechanics and its Applications, vol. 53, pp. 151-158. Springer (1999)

2. Janke, E.: Receptivity of three-dimensional boundary layers to roughness and suction - Linear and nonlinear effects. In: Wagner, S., Rist, U., Heinemann, H.J., Hilbig, R. (eds.) New Results in Numerical and Experimental Fluid Mechanics III. Notes on Numerical Fluid Mechanics (NNFM), vol. 77, pp. 367-374. Springer (2002). https://doi.org/10.1007/978-3-540-45466-3_43

3. Hill, D.C.: Adjoint systems and their role in the receptivity problem for boundary layers. J. Fluid Mech. 292, 183-204 (1995)

4. Hill, D.C.: Receptivity in non-parallel boundary layers. In: Proceedings of the 1997 ASME Fluids Engineering Division Summer Meeting, FEDSM97-3108 (1997)

5. Pralits, J.O., Airiau, C., Hanifi, A., Henningson, D.S.: Sensitivity analysis using adjoint parabolized stability equations for compressible flows. Flow Turbulence Combustion 65, 321-346 (2000)

6. Dobrinsky, A.: Adjoint analysis for receptivity prediction. Ph.D. thesis, Rice University (2002)

7. Tempelmann, D.: Receptivity of crossflow-dominated boundary layers. Ph.D. thesis, Royal Institute of Technology Stockholm (2011)

8. Schrader, L.U.: Receptivity of boundary layers under pressure gradient. Licentiate thesis, Royal Institute of Technology Stockholm (2008)

9. Piot, E., Schrader, L.U.: Receptivity of three-dimensional boundary layers to surface roughness. ERCOFTAC Bull. 74, 13-19 (2007)

10. Bertolotti, F.P.: Receptivity of three-dimensional boundary-layers to localized wall roughness and suction. Phys. Fluids 12, 1799-1809 (2000) 
11. Hein, S., Bertolotti, F.P., Simen, M., Hanifi, A., Henningson, D.: Linear nonlocal instability analysis - the linear NOLOT code. DLR Internal Report, DLR IB 22394, A56 (1995)

12. Schrauf, G.H., von Geyr, H.: Simplified hybrid laminar flow control for the A320 fin - Aerodynamic and system design, first results. AIAA Paper 2020-1536 (2020)

13. Schrauf, G. H.: COCO - a program to compute velocity and temperature profiles for local and nonlocal stability analysis of compressible, conical boundary layers with suction. ZARM Technik Report, November 1998

14. Crouch, J.: Receptivity of three-dimensional boundary layers. AIAA Paper 19930074 (1993)

15. Tempelmann, D., Hanifi, A., Hein, S., Henningson, D. S.: Validation of receptivity models: comparison with KTH wind tunnel tests. TELFONA (Testing for laminar flow on new aircraft), Deliverable No. D4.2-5 (2009) 